\title{
MicroRNA-206 contributes to the progression of steroid-induced avascular necrosis of the femoral head by inducing osteoblast apoptosis by suppressing programmed cell death 4
}

\author{
ZAIHENG ZHANG $^{1}$, ANMIN JIN ${ }^{2}$ and DENGLU YAN ${ }^{3}$ \\ ${ }^{1}$ Department of Orthopedics, Baoan People's Hospital of Southern Medical University, Shenzhen, Guangdong 518101; \\ ${ }^{2}$ Department of Orthopedics, Zhujiang Hospital of Southern Medical University, Guangzhou, Guangdong 510280; ${ }^{3}$ Department of \\ Orthopedics, People's Hospital of Nanshan District, Shenzhen, Guangdong 518000, P.R. China
}

Received May 13, 2017; Accepted September 15, 2017

DOI: $10.3892 / \mathrm{mmr} .2017 .7963$

\begin{abstract}
The expression of microRNA-206 (miR-206) is aberrantly induced in steroid-induced avascular necrosis of femoral head (SANFH). Therefore, investigating the function of miR-206 in SANFH and uncovering the functional mechanism associated with the condition will promote the understanding and treatment of the disease. The purpose of the present study was to investigate the pro-osteoclasteogenic effect of miR-206 that occurs through regulation of programmed cell death 4 (PDCD4). The expression of miR-206 and PDCD4 was analyzed in the clinical SANFH specimens. The level of miR-206 and PDCD4 was regulated in human osteoblast lineage hFOB1.19 and the effect of different treatments on cell viability, proliferation, apoptosis and differentiation potential of osteoblasts were analyzed with a Cell Counting kit-8, 5-ethynyl-2'-deoxyuridine staining, flow cytometry and Hoechst staining. The expression of miR-206 was upregulated while PDCD4 was downregulated in the SANFH specimens. Induced expression of miR-206 decreased cell viability and proliferation, while apoptosis was induced. At the molecular level, overexpression of miR-206 inhibited the expression of PDCD4, alkaline phosphatase (ALP) and B-cell lymphoma 2 (Bcl-2), and increased the expression of apoptosis regulator $\mathrm{Bcl} 2-\mathrm{X}$-associated protein (Bax). Inhibiting the expression of miR-206 increased cell viability and proliferation but had no effect on cell apoptosis, as detected by flow cytometry and Hoechst staining. However, at the molecular level, inhibiting the expression of miR-206 induced expression of PDCD4, ALP and Bcl-2, while it decreased the expression of Bax. Additionally, knockdown of PDCD4 blocked the effect of miR-206 inhibition on hFOB1.19 cells, representing a
\end{abstract}

Correspondence to: Dr Anmin Jin, Department of Orthopedics, Zhujiang Hospital of Southern Medical University, 253 Gongye Road, Guangzhou, Guangdong 510280, P.R. China

E-mail: jinanmin988@163.com

Key words: apoptosis, microRNA 206, osteoblast, programmed cell death 4, steroid-induced avascular necrosis of femoral head
PDCD4-dependent manner of miR-206 in inducing apoptosis of osteoblasts. Therefore, miR-206 promoted the onset of SANFH by inducing apoptosis and suppressed the proliferation of osteoblasts, which was dependent on the inhibition of PDCD4.

\section{Introduction}

Perthes' disease is a self-limiting disease of children, and causes interrupted blood supply to the capital femoral epiphysis (1). As one of the major outcomes of the deficient blood supply associated with Perthes' disease, a large proportion of the patients will end up with steroid-induced avascular necrosis of femoral head (SANFH), which is characterized by degeneration of the femoral head and death of the dynamics component of bone (2). Since the 1960s, scientists have been attempting to reveal the pathogenesis of SANFH and have proposed multiple theories. Mechanisms including fat coagulation, increased intraosseous pressure and osteoporosis have been investigated for their potential to identify the pathogenesis of SANFH $(3,4)$. However, even with the investigation of SANFH, there are a number of questions that need to be answered concerning the pathological alterations associated with osteocytes and osteoblasts in SANFH. In previous years, several studies have inferred the strong association of osteoblastogenesis with SANFH $(5,6)$. A comprehensive understanding of the pattern of the death of osteocytes and osteoblasts, and factors involved in the process, will provide a novel insight into the pathogenesis of SANFH. Several studies have already been performed to validate the key role of the fate of osteoblasts in determining the onset of $\operatorname{SANFH}(7,8)$. It is also been demonstrated that a deficiency in the number and function of osteoblasts results in impaired osteogenic capacity, and therapies based on osteoblast grafts can alleviate the symptom of femoral head necrosis (9). Therefore, identification of biomarkers predicating or modulating the proliferation of osteoblasts will open novel therapeutic avenues for SANFH.

MicroRNAs (miRs) have important roles in determining the growth, differentiation and function of bone cells (10). By binding to $3^{\prime}$ untranslated regions (3'UTRs) of target mRNAs, miR family members can repress the function of the targeted 
genes. More than $600 \mathrm{miR}$ members have been proved to have an effect on bone and cartilaginous tissues, influencing the fate of osteoblasts and osteoclasts as well as chondrocytes and other mesenchymal cell types (11-15). Inose et al (16) demonstrated that the level of miR-206 is markedly downregulated during differentiation of $\mathrm{C} 2 \mathrm{C} 12$ cells into osteoblasts, while overexpression of miR impairs bone formation by targeting the gap junction a-1 protein $(9,16)$. In addition, the association between the effect of miR-206 on osteogenic differentiation with the onset of SANFH is further explained by Liu et al (17), by connecting the function of the miR to the Wnt/ $\beta$-catenin signaling pathway. Results of the two studies confirmed the crucial role of miR-206 in determining the differentiation potential of osteoblasts. Therefore, it is reasonable to investigate the signaling pathways involved in the function of miR-206 in osteoblasts.

Although hundreds of targets of miR-206 have been predicated by bioinformatics analysis, the present study focused on the expression of programmed cell death 4 (PDCD4) in osteoblasts. Expression of the PDCD4 gene influences the activity of the transcription factor AP-1 directly $(18,19)$, and diminished PDCD4 level allows initiation of the osteoclastogenic program by releasing proto oncogene c-Fos from inhibition (10). Given the function of miR-206 and PDCD4 in bone metabolic processes, a clear explanation of the interaction between the two factors will further highlight the process underlying the pathological alterations of SANFH. In the present study, the expression status of miR-206 and PDCD4 were first investigated with clinical SANFH samples. Subsequently, the expression levels of miR-206 and PDCD4 were modulated to assess their exact roles in the proliferation and apoptosis of osteoblasts. The results of the present study suggested that miR-206 promoted the onset of SANFH, by inducing osteoblast apoptosis via inhibition of PDCD4.

\section{Materials and methods}

Chemicals and agents. Antibodies against PDCD4 (cat. no. ab45263), alkaline phosphatase (ALP; cat. no. ab83259), Bcl-2-associated X protein (Bax; cat. no. ab32503) and B-cell lymphoma 2 (Bcl-2; cat. no. ab32124) were purchased from Abcam (Cambridge, UK). The antibody against GAPDH (KC-5G5) was purchased from Zhejiang Kangchen Biotech Co., Ltd. (Wuhan, China). The secondary goat anti-rabbit (cat. no. BA1054) immunoglobulin (Ig)G-horseradish peroxidase-conjugated antibody was purchased from Boster Biological Technology (Pleasanton, CA, USA). A mimic (5'-UGGAAU GUAAGGAAGUGUGUGG-3') and inhibitor (5'-CCACAC ACUUCCUUACAUUCCA-3') for miR-206 was obtained from Chang Jing Bio-Tech, Ltd. (Changsha, China). A normal control (NC) mimic (5'-UUCUCCGAACGUGUCACGUT-3') was purchased from Chang Jing Bio-Tech, Ltd. The Annexin V/propidium iodide (PI) apoptosis kit (cat. no. CCS012) was purchased from MultiSciences Biotech Co., Ltd. (Susteren, The Netherlands). Lipofectamine ${ }^{\mathrm{TM}} 2000$ (cat. no. 52887) reagent was obtained from Invitrogen; Thermo Fisher Scientific, Inc. (Waltham, MA, USA). The Cell-Light ${ }^{\mathrm{TM}}$ 5-ethynyl-2'-deoxyuridine (EdU) Apollo ${ }^{\circledR} 488 / 567$ in vitro Imaging kit was purchased from Guangzhou RiboBio Co., Ltd. (Guangzhou, China; cat. no. C10327). RNAiso Plus (cat. no. 9109) was from Takara Bio, Inc. (Otsu, Japan). The Reverse Transcription (RT) kit and quantitative polymerase chain reaction (qPCR) agents were purchased from DBI ${ }^{\circledR}$ Bioscience (www.xinghanbio.com/). A protein Concentration Determination kit (cat. no. 23227) was purchased from Thermo Fisher Scientific, Inc. A Dual Luciferase Assay kit (cat. no. E1980) was purchased from Promega Corporation (Madison, WI, USA).

Cell cultures. Human osteoblast lineage hFOB1.19 and 293T cells were purchased from the American Type Culture Collection (Manassas, VA, USA) and maintained in media consisting of $45 \%$ Dulbecco's modified Eagle's medium (Gibco; Thermo Fisher Scientific, Inc.), 45\% F12 medium (Gibco; Thermo Fisher Scientific, Inc.), $10 \%$ fetal bovine serum and $1 \%$ mixed antibiotics (v/v) (penicillin/streptomycin) (R\&D Systems, Inc., Minneapolis, MN, USA) at $37^{\circ} \mathrm{C}$ in an atmosphere of $5 \% \mathrm{CO}_{2}$ and $95 \%$ air. Cells from passage three to five were used for assays in the present study.

Specimen collection. SANFH specimens were collected from 15 SANFH patients from February 2015 to March 2017 in the Department of Orthopedic Pathology in Baoan People's Hospital of the Southern Medical University (Shenzhen, China). A total of 15 bone specimens from patients undergoing total hip replacement for femoral neck fracture were collected as controls. All the cases involved in the present study had detailed information on the clinicopathological and prognostic characteristics. The present study was approved by Baoan People's Hospital of the Southern Medical University Ethics Committee. The Ethics Committee approved the associated screening, inspection and data collection of the patients, and all subjects signed a written informed consent form. All experiments were undertaken following the provisions of the Declaration of Helsinki.

Plasmid construction and transfection. Specific small interfering (si)RNA (50 nM) targeting PDCD4 (5'-UCAGUAUCU GCUCAUUUUCUATT-3') and negative control (NC) siRNA (50 nM) (5'-UUCUCCGAACGUGUCACGUTT-3') were purchased from Chang Jing Bio-Tech, Ltd. For the dual luciferase assay, wild type and mutant sequences of PDCD4 3'UTR were synthesized by Sangon Biotech Co., Ltd. (Shanghai, China) and ligated into the psiCHECK-2 plasmid (Promega Corporation, Madison, WI, USA). Transfection was performed using Lipofectamine ${ }^{\mathrm{TM}} 2000$ according to the manufacturer's protocol. Twenty-four hours after transfection, cells were collected and subjected to subsequent experiments.

$R N A$ extraction and $R T-q P C R$. Total RNA from SANFH specimens or hFOB1.19 cells (normal, NC siRNA transfected, and $P D C D 4$ siRNA transfected) was extracted using an RNA extraction kit according to the manufacturers' protocol. cDNA templates were obtained via RT using RNA according to the manufacturer's protocol. The final reaction mixture of volume $20 \mu \mathrm{l}$ contained $10 \mu \mathrm{l}$ Bestar ${ }^{\circledR}$ SYBR-Green qPCR Master Mix and $0.5 \mu \mathrm{l}$ each primer. PDCD4 forward, 5'-ACAGGTGTA TGATGTGGAGGA-3' and reverse, 5'-TTCTCAAATGCC CTTTCATCCAA-3'; miR-206 forward, 5'-ACACTCCAG CTGGGTGGAATGTAAGGAAGTGT-3' and reverse, 5'-CTC AACTGGTGTCGTGGA-3'; ALP forward, 5'-ACTGGGGCC TGAGATACCC-3' and reverse, 5'-TCGTGTTGCACTGGT TAAAGC-3'. GAPDH was used as an internal reference, the 
primers were as follows: Forward, 5'-ATCGTGCGTGAC ATTAAGGAGAAG-3' and reverse, 5'-AGGAAGGAAGGC TGGAAGAGTG-3'. U6 was used as an internal reference, the primers were as follows: Forward, 5'-CTCGCTTCGGCAGCA CA-3' and reverse, 5'-AACGCTTCACGAATTTGCGT-3'. A total of $1 \mu \mathrm{l}$ cDNA template and $8 \mu \mathrm{l}$ RNase free $\mathrm{H}_{2} \mathrm{O}$ reaction volume was used. The thermocycling conditions for the amplification were as follows: A denaturation step at $94^{\circ} \mathrm{C}$ for $2 \mathrm{~min}$, followed by 40 cycles at $94^{\circ} \mathrm{C}$ for $20 \mathrm{sec}, 58^{\circ} \mathrm{C}$ for $20 \mathrm{sec}$ and $72^{\circ} \mathrm{C}$ for $30 \mathrm{sec}$, and the reaction was stopped by a step at $25^{\circ} \mathrm{C}$ for $5 \mathrm{~min}$. The melting curve was analyzed between $62^{\circ} \mathrm{C}$ and $95^{\circ} \mathrm{C}$, and the relative expression levels of the target genes were calculated by a Real-Time PCR Detection system (Mx3000P; Agilent Technologies, Inc., Santa Clara, CA, USA) using the formula of $2^{-\Delta \Delta C q}(20)$.

Western blot assay. Total proteins were extracted using the Total Protein Extraction kit according to the manufacturer's protocol. The concentrations of the protein samples were determined according to the Bicinchoninic Acid assay. $30 \mu \mathrm{g}$ protein from different samples were subjected to $10 \%$ sodium dodecyl sulfate polyacrylamide gel electrophoresis at $80 \mathrm{~V}$ for $2.5 \mathrm{~h}$. The proteins were transferred onto polyvinylidene difluoride membranes and rinsed with Tris base buffer solution with Tween-20 (TBST). Following this, membranes were blocked with skimmed milk solution for $1 \mathrm{~h}$ at room temperature. Subsequently, membranes were incubated with one of the primary antibodies against PDCD4 $(1: 2,000)$ for $50 \mathrm{~min}$, anti-Bax $(1: 1,000)$ for $25 \mathrm{~min}$, anti-ALP $(1: 1,000)$ for $40 \mathrm{~min}$, anti-Bcl-2 $(1: 1,000)$ for $25 \mathrm{~min}$, and anti-GAPDH (internal reference; 1:1,000) for $30 \mathrm{~min}$ at $300 \mathrm{~mA}$ at room temperature and then incubated with peroxidase-conjugated secondary antibodies $(1: 20,000)$ for $40 \mathrm{~min}$ at room temperature. Blots were developed using the Beyo ECL Plus reagent (Beyotime Institute of Biotechnology, Haimen, China) and analyzed with the Gel Imaging system (WD-9413B; Beijing Liuyi Biotechnology Co., Ltd., Beijing, China) and the relative expression levels of different proteins were calculated with Bio-Rad Quantity One software version 4.6.3 (Bio-Rad Laboratories, Inc., Hercules, CA, USA).

Dual luciferase assay. The modulation effect of miR-206 on PDCD4 was determined with dual luciferase assay in $293 \mathrm{~T}$ cells $\left(2 \times 10^{5} / \mathrm{ml}\right)$ : luciferase activity was detected by Dual Luciferase Assay kit according to the manufacturers' instruction $48 \mathrm{~h}$ following transfection of different combinations of plasmid and mimic. psi-CHECK2-PDCD4 and miR-206 mimic co-transfection was performed with the with Lipofectamine 2000. The fluorescence intensity was detected using a Microplate Reader (GloMax; Promega Corporation) and normalized in comparison with Renilla luciferase.

Cell viability and proliferation detection. Exponentially growing hFOB1.19 cells $\left(5 \times 10^{5}\right.$ cells $\left./ \mathrm{ml}\right)$ were seeded into a 96-well plate. Then cells were incubated for $72 \mathrm{~h}$ (each treatment was administered nine times) and every $24 \mathrm{~h}$, $10 \mu \mathrm{l} / \mathrm{ml}$ CCK-8 (Beyotime Insitute of Biotechnology) solution was added to selected wells and incubated at $37^{\circ} \mathrm{C}$ for $60 \mathrm{~min}$. Optical density values at $450 \mathrm{~nm}$ were measured with a microplate reader (GloMax; Promega Corporation) and used to give a representative assessment of cell viability. hFOB1.19 cells were harvested following a 48-h culture and assessed using 5-ethynyl-2'-deoxyuridine (EdU) assay according to the manufacturer's protocol and the result was detected under a fluorescent microscope, magnification, 200x.

Immunofluorescence detection. Immunofluorescence detection was conducted as described in a previous study (21). Briefly, paraformaldehyde-fixed cells were permeabilized with $0.1 \%$ Triton X-100 for $30 \mathrm{~min}$. Following blocking in 10\% goat serum (Thermo Fisher Scientific, Inc.) for 15 min, primary rabbit polyclonal antibodies against different proteins of interest (PDCD4) (1:500) were then added, and the cells were incubated overnight at $4^{\circ} \mathrm{C}$. Subsequently, cells were incubated with $\mathrm{Cy} 3$-labeled secondary antibody $(1: 1,000)$ (cat. no. ab6939; Abcam) for $1 \mathrm{~h}$ in the dark. Thereafter, the cells were stained with 4,6-diamino-2-phenyl indole for $5 \mathrm{~min}$ at room temperature. The results were imaged with a fluorescent microscope (BX53; Olympus Corporation, Tokyo, Japan) at magnification, x40.

Hoechst 33258 staining. The morphological alterations of the cellular nuclei due to apoptosis were detected using a Hoechst staining kit $(20 \mu \mathrm{M})$ according to the manufacturer's protocol. The results were observed under a fluorescence microscope under $460 \mathrm{~nm}$; magnification, x120.

Flow cytometry. Apoptotic process was detected using an Annexin V/PI apoptosis kit according to the manufacturer's protocol. The apoptotic rates were analyzed using a FACScan flow cytometer (Accuri C6; BD Biosciences). The total apoptotic rate was equal to the sum of the late apoptotic rate (UR, upper right quadrant-advanced stage apoptosis) and the early apoptotic rate (LR, lower right quadrant-prophase apoptosis).

Statistical analysis. All the data were expressed as the mean \pm the standard deviation $(n=3)$. The association between expression of miR-206 and PDCD4 at mRNA level was analyzed with Pearson correlation analysis. The difference between groups was analyzed using Student's t-test or one-way analysis of variance followed by the Least Significant Difference. All the statistical analyses were conducted using SPSS version 19.0 (IBM Corp., Armonk, NY, USA). P<0.05 was considered to indicate a statistically significant difference.

\section{Results}

Expression level of miR-206 increases while expression of PDCD4 is suppressed in SANFH samples. A total of 15 SANFH samples were collected to investigate the association between the expression of miR-206 and PDCD4. As presented in Fig. 1A, the expression of miR-206 was significantly increased in SANFH samples compared with the control samples $(\mathrm{P}<0.001)$. However, the expression of PDCD4 was significantly inhibited at the mRNA and protein level $(\mathrm{P}<0.01$; Fig. 1B and C, respectively). In addition, it was demonstrated that miR-206 was negatively correlated with PDCD4 at the mRNA level $(R=0.633, P<0.01$; Fig. 1D), indicating a potential interaction between the two factors.

miR-206 directly binds to the promoter of the PDCD4 gene and inhibits the transcription of PDCD4. The interaction 
A

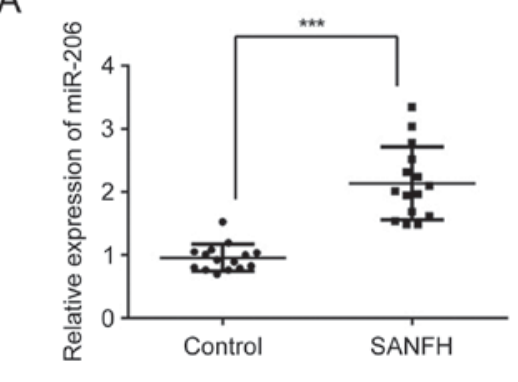

C
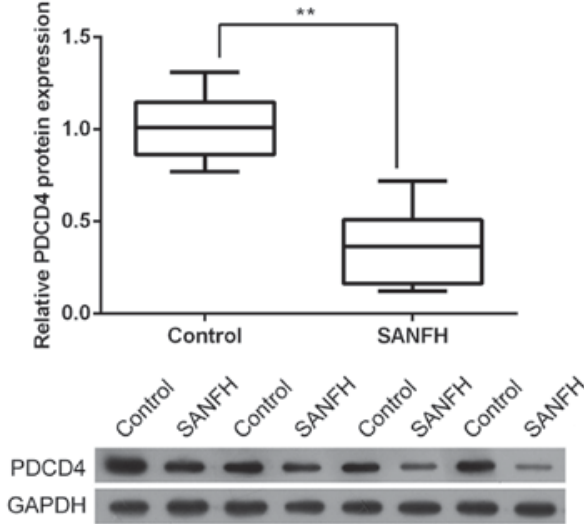

$\mathrm{B}$

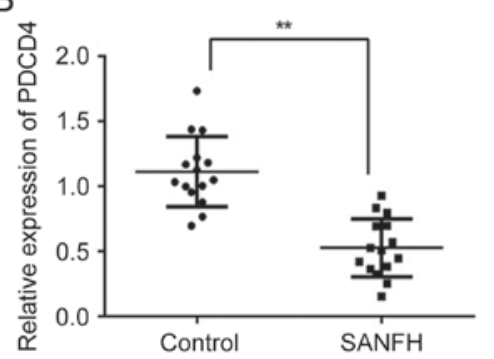

D

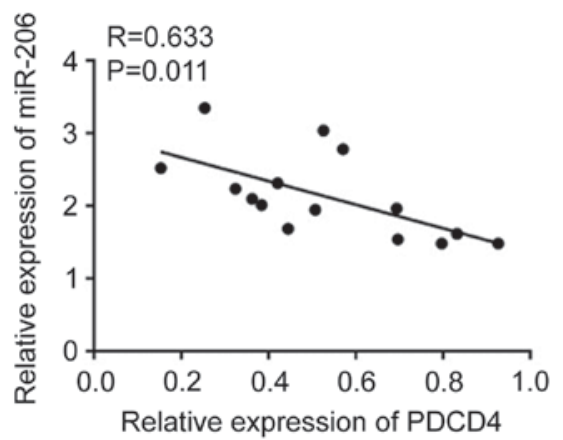

Figure 1. Analysis of the expression of miR-206 and PDCD4 in the SANFH specimens. Investigation using RT-qPCR demonstrated that the (A) mRNA expression level of miR-206 was increased compared with the control, while (B) RT-qPCR and (C) western blotting demonstrated that PDCD4 was inhibited in SANFH specimens compared with the control. (D) Correlation analysis between the relative expression of PDCDA and miR-206. There was a negative correlation between the expression levels of the two indicators. Data are presented as the mean \pm standard deviation. ${ }^{* *} \mathrm{P}<0.01$ and ${ }^{* * *} \mathrm{P}<0.001 \mathrm{vs}$. control. $\mathrm{RT}-\mathrm{qPCR}$, reverse transcription-quantitative polymerase chain reaction; miR, microRNA; PDCD4, programmed cell death 4; SANFH, steroid-induced avascular necrosis of the femoral head.

between miR-206 and PDCD4 was analyzed with a dual luciferase assay. As presented in Fig. 2, the co-transfection of miR-206 and the wild type promoter sequence of PDCD4 significantly decreased the relative luciferase activity in 293T cells $(\mathrm{P}<0.05)$. Given that miR-206 and the mutant promoter sequence of PDCD4 had no effect on relative luciferase activity, the results of the dual luciferase assay indicated the that miR-206 bound directly and specifically to the promoter sequence of the PDCD4 gene, which exerted a negative effect on the expression of PDCD4 at the transcriptional level.

miR-206 contributes to suppressing cell viability and proliferation, and inducing apoptosis in hFOB1.19 cells. The function of miR-206 in osteoblastogenesis was assessed by altering the expression of miR-206 in a bilateral pattern. Both a specific mimic and inhibitor of miR-206 were stably introduced into the human osteoblast lineage hFOB1.19. Based on the results of the CCK-8 assay, the cell viability of miR-206 overexpressing cells was significantly decreased, while the viability of the cells treated with an miR-206 inhibitor significantly increased compared with the control $(\mathrm{P}<0.05$; Fig. 3A). Consistent with the results of the CCK- 8 assay, a greater number of EdU positive cells (stained red) were detected in the cells treated with the miR-206 inhibitor while fewer was detected with miR-206 overexpressing cells (Fig. 3B). The cells overexpressing-miR-206 demonstrated a significantly increased level of apoptosis as illustrated by Hoechst 33258 staining and flow cytometry: A larger proportion of Hoechst33258 positive cells (bright blue), and significantly increased apoptotic rate were detected $(\mathrm{P}<0.01$; Fig. 3C and D). However, inhibition of miR-206 in hFOB1.19

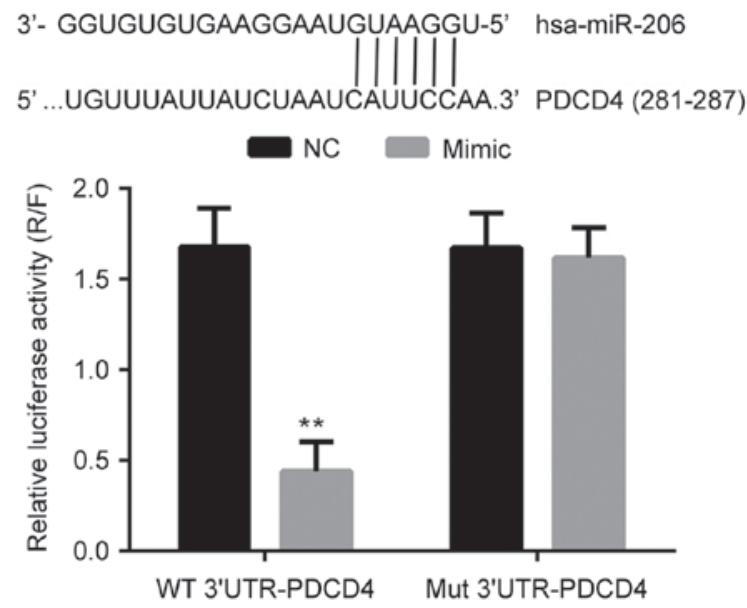

Figure 2. Complementary sequences of hsa-miR-206 and PDCD4 and results of a dual luciferase assay demonstrating direct binding of miR-206 to the promoter sequence of PDCD4 gene. Data are presented as the mean \pm standard deviation. ${ }^{* *} \mathrm{P}<0.01$ vs. NC. NC, negative control; miR, microRNA; PDCD4, programmed cell death 4; WT, wild type; Mut, mutant; UTR, untranslated region. $\mathrm{R} / \mathrm{F}$, Renilla to firefly activity ratio.

cells had no influence on the cell apoptotic process compared with the NC group (Fig. 3C and D).

miR-206 impairs the osteogenic process by suppressing PDCD4-associated signaling. At the molecular level, transfection of an miR-206 mimic downregulated the expression of PCDCD4 (Fig. 4A-C). Additionally, ALP was also suppressed by transfection with an miR-206 mimic (Fig. 4A 
A

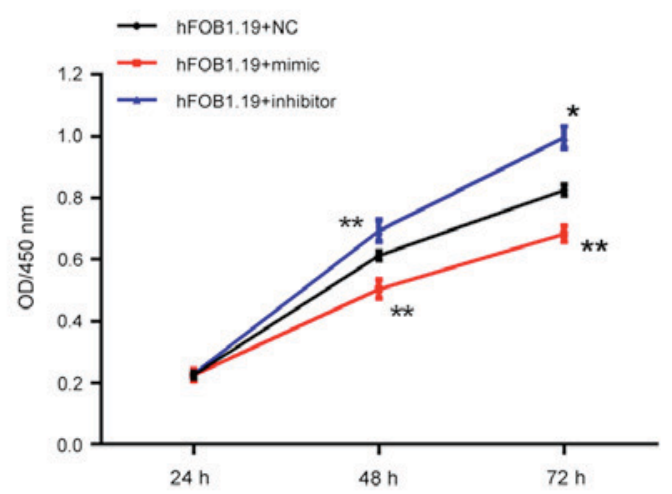

C
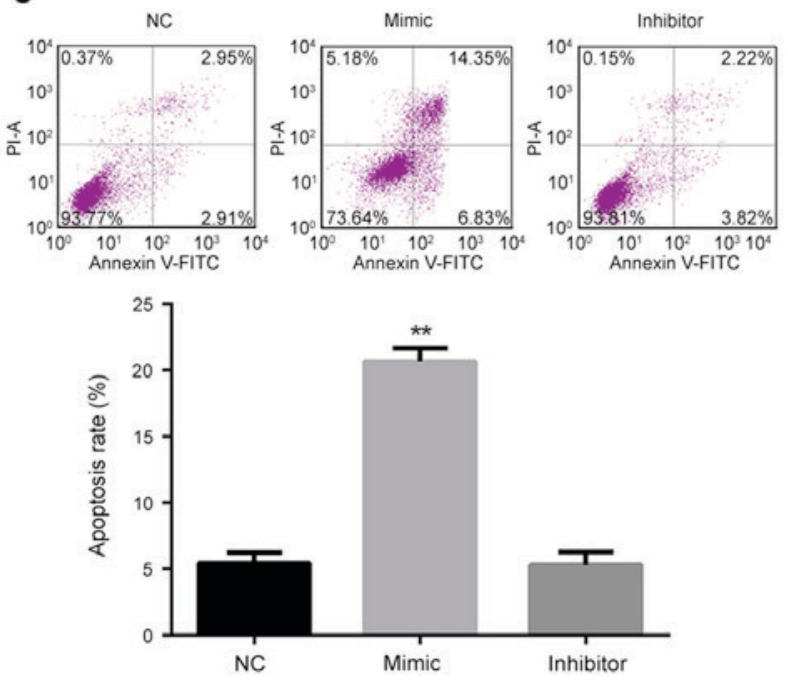

B
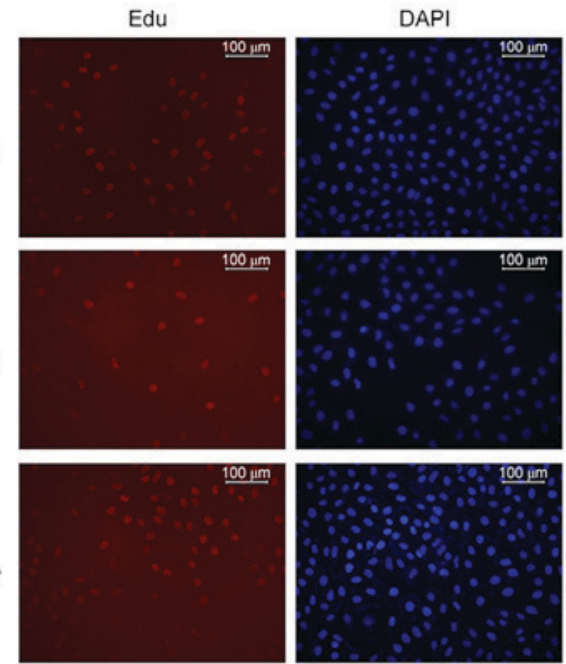

D

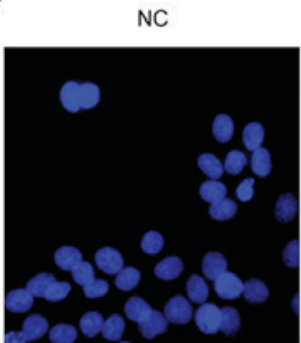

Mimic

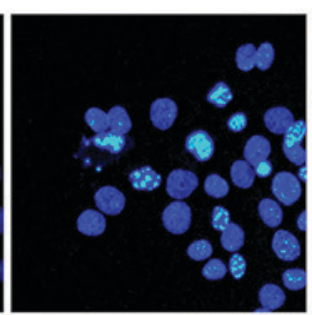

Merge
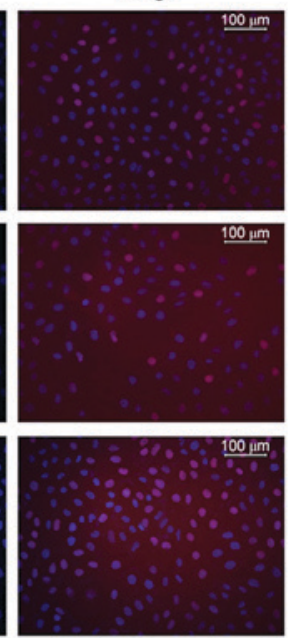

Figure 3. Analysis of the effect of regulation of miR-206 on cell viability, proliferation and apoptosis in hFOB1.19 cells. (A) Cell viability as detected by the Cell Counting kit- 8 assay was decreased by the miR-206 mimic and increased by the miR-206 inhibitor. (B) Cell proliferation as detected by EdU assay demonstrated that proliferation of hFOB1.19 cells was inhibited by the miR-206 mimic and induced by the miR-206 inhibitor. Proliferating cells were stained red by EdU. (C) Cell apoptosis detected by flow cytometry and (D) Hoechst staining demonstrated that the miR-206 mimic induced apoptosis and while miR-206 inhibitor had no influence on apoptosis in hFOB1.19 cells. " $\mathrm{P}<0.05$ vs. NC. ${ }^{* *} \mathrm{P}<0.01$ vs. NC. miR, microRNA; EdU, 5-ethynyl-2'-deoxyuridine; NC, negative control; FITC, fluorescein isothiocyanate; PI, propidium iodide; OD, optical density; DAPI, DAPI, 4,6-diamino-2-phenylindole.

and $\mathrm{B}$ ), indicating the impaired proliferation and differentiation potential of hFOB1.19 cells as a result of miR-206 overexpression. The level of the pro-apoptotic factor Bax was increased while the level of anti-apoptosis factor Bcl-2 was suppressed in miR-206 overexpressing cells (Fig. 4B). For cells transfected with the miR-206 inhibitor, the expression of Bax was decreased and the expression of PCDCD4 and Bcl-2 was increased (Fig. 4B and C), representing an inhibited apoptotic process in hFOB1.19 cells, which explained the augmented cell viability by miR-206 knockdown.

The involvement of PDCD4 in osteoblastogenesis had been previously reported (22), but the exact function of the factor on bone metabolism was neglected. The effect of PDCD4 and miR-206 on the differentiation potential, and apoptosis was investigated (Fig. 5). In the present study, the expression of PDCD4 was inhibited in miR-206 knockdown hFOB1.19 cells, which resulted in significantly decreased expression of ALP $(\mathrm{P}<0.001$; Fig. 5A). It was demonstrated that the knockdown of PDCD4 significantly induced cellular apoptosis in a similar pattern to that of miR-206 overexpression ( $\mathrm{P}<0.01$; Fig. $5 \mathrm{~B}$ and D). In addition, knockdown of PDCD4 induced the expression of Bax while the expression of ALP and Bcl-2 was suppressed (Fig. 5C), which relieved the hFOB1.19 cells of the effect of miR-206 inhibition. The above results indicated that the pro-SANFH function of miR-206 depended on the suppression of PDCD4 in osteoblasts.

\section{Discussion}

The incidence of SANFH has continued to increase due to the excessive use of exogenous steroids in recent years (17). Although numerous studies have attempted to investigate the molecular alterations associated with the disease and develop novel treatment or prevention modalities, the onset and progression mechanism underlying $\mathrm{SANFH}$ remain partially understood. Based on clinical statistics, the prognosis of SANFH children is significantly better than that of adults (23-25). Given that children possess a stronger osteogenic ability than adults, the viability and differentiation potential of osteoblasts is potentially serving a key role in the onset of SANFH. Therefore, the identification of biological markers indicating the proliferation/apoptosis of osteoblasts 
A
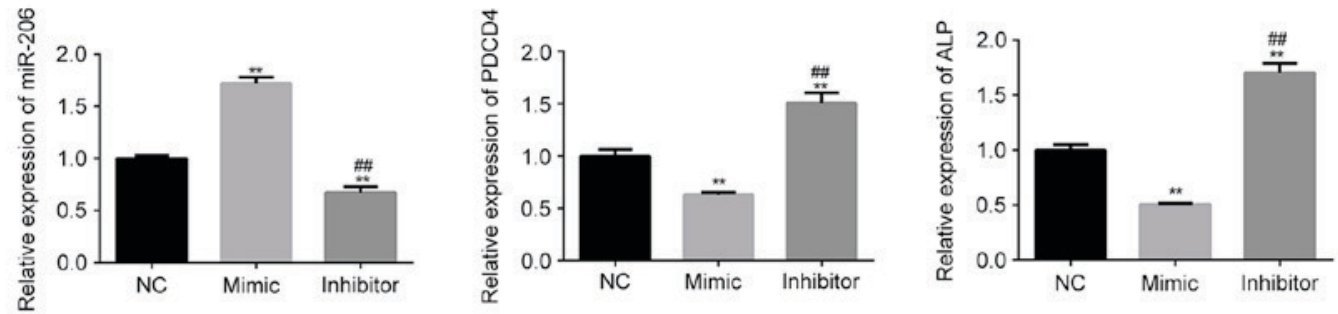

B
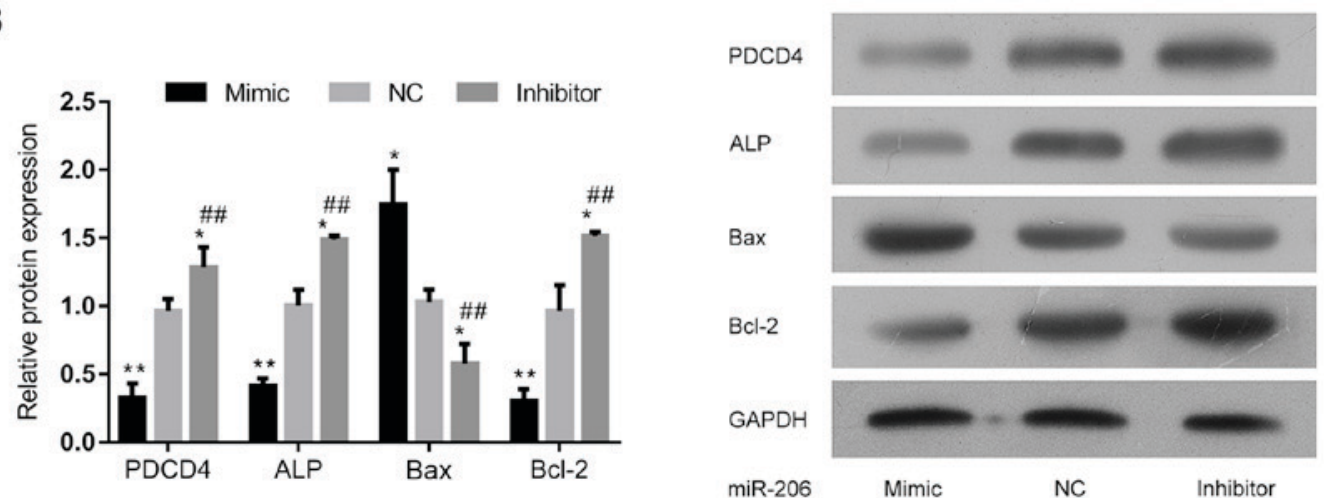

C
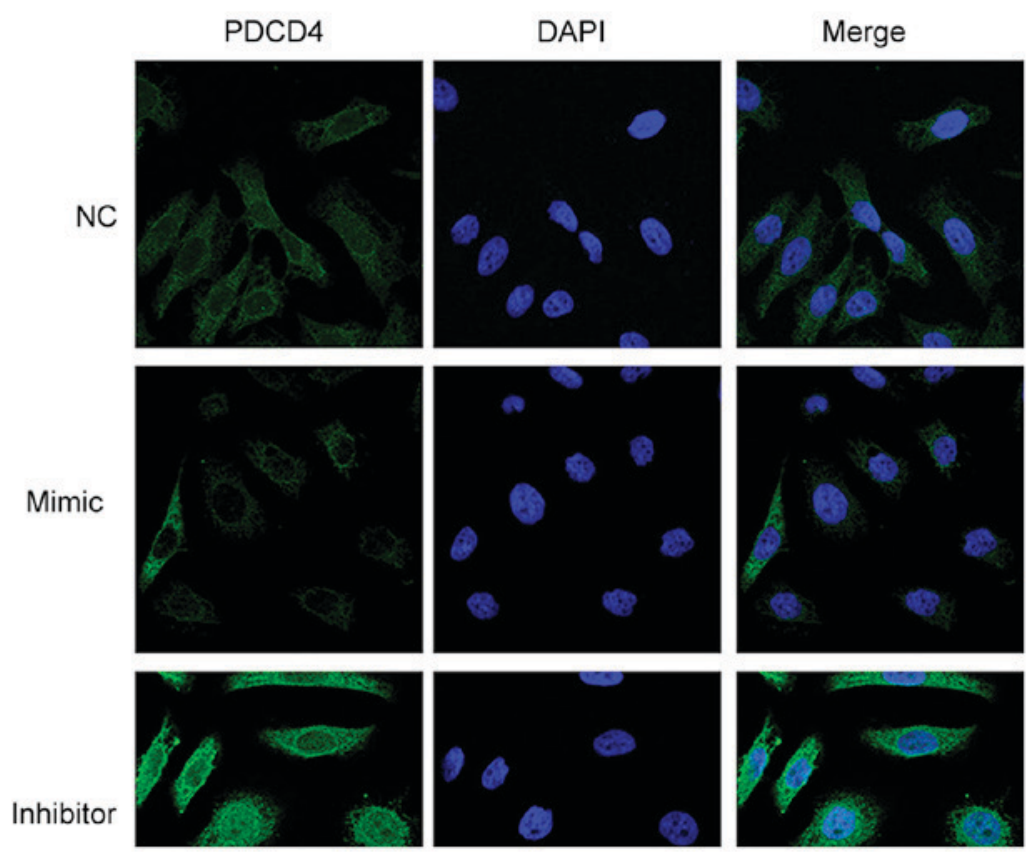

Figure 4. Regulation of miR-206 influences the expression of the different signaling molecules associated with differentiation and apoptosis in hFOB1.19 cells. (A) Relative expression of miR-206, PDCD4 and ALP in hFOB1.19 cells transfected with an miR-206 inhibitor, mimic or NC. (B) Western blotting demonstrating the expression of PDCD4, ALP, Bcl-2, Bax and GAPDH in hFOB1.19 cells transfected with an miR-206 inhibitor, mimic or NC. (C) Transfection of miR-206 mimic suppressed the expression of PDCD4. (D) Transfection of miR-206 inhibitor induced the expression of PDCD4. "P<0.05, " $\mathrm{P}<0.01$ vs. NC. ${ }^{\# \#} \mathrm{P}<0.01$ vs. mimic. PDCD4, programmed cell death 4; ALP, alkaline phosphatase; Bcl-2, B-cell lymphoma 2; Bax, Bcl-2-X-associated protein; miR, microRNA; NC, negative control; DAPI, 4,6-diamino-2-phenylindole.

is a reasonable target for analysis to promote improvement in the treatment and prevention of SANFH. In the present study, the results demonstrated that the apoptotic process of human osteoblast lineage hFOB1.19 was governed by miR-206 in a PDCD4-inhibition-dependent manner.

The integrity and function of the skeletal system is maintained by a balance between osteoclasts and osteoblasts, interruption of this during any stage of life will lead to bone disease, such as osteonecrosis (26). Based on several previous studies, the apoptosis of osteoblasts is a critical event in the onset of SANFH as the process disrupts the balance between osteoclasts and osteoblasts $(7,8,27)$. Multiple factors are reported to be associated with the viability and survival of osteoblasts. Among these, miRs have emerged as novel regulatory molecules closely associated with bone diseases (17). A number of miRs exhibit a tissue or developmental stage-specific expression pattern, and have been depicted as signaling network nodes modulating osteoblastic differentiation processes. For example, miR-21, 

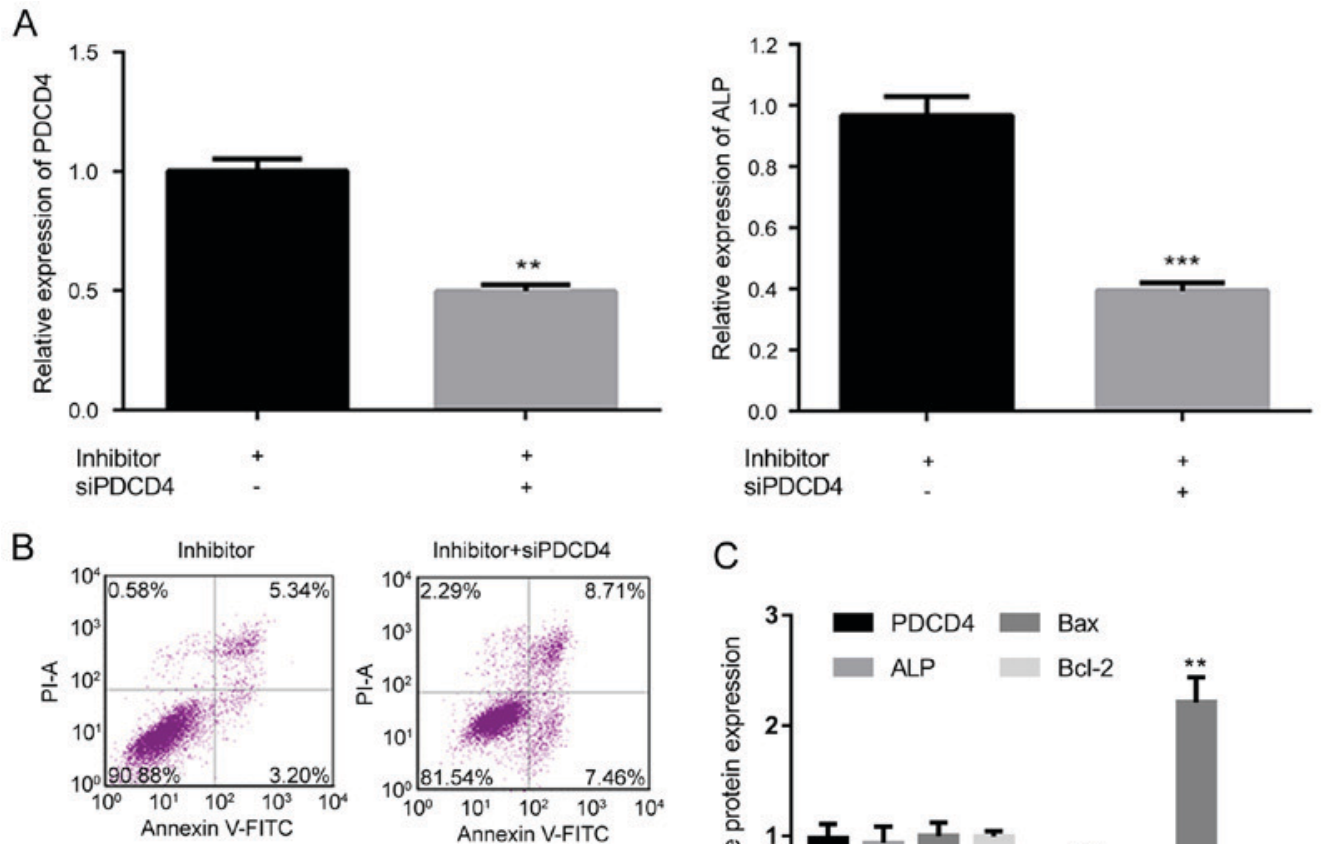

C
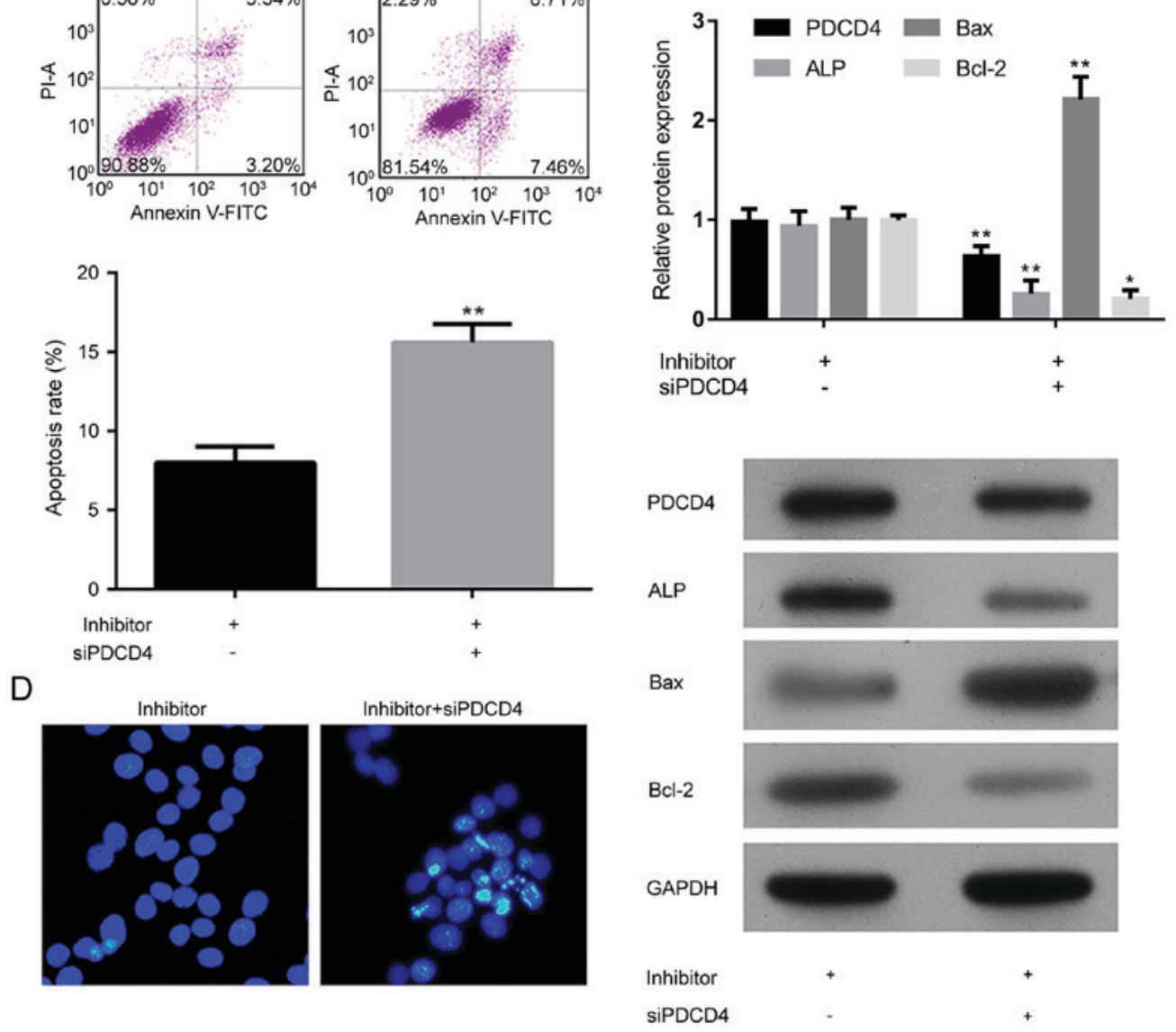

Figure 5. Analysis of the effect of miR-206 on the differentiation potential and apoptosis in hFOB1.19 cells depended on the suppression of PDCD4 associated signaling. Analysis of the relative expression of (A) PDCD4 and ALP in hFOB1.19 cells transfected with the miR-206 inhibitor and an siRNA against PDCD4. (B) Cell apoptosis and quantification, detected by flow cytometry of cells transfected with miR-206 inhibitor and an siRNA against PDCD4. (C) Western blotting and quantification demonstrating the expression of PDCD4, ALP, Bcl-2 and Bax, in hFOB1.19 cells transfected with miR-206 inhibitor and an siRNA against PDCD4. (D) Hoechest staining of hFOB1.19 cells transfected with the miR-206 inhibitor and an siRNA against PDCD4. Data are expressed as the mean \pm standard deviation. "P $<0.05,{ }^{* * *} \mathrm{P}<0.01,{ }^{* * *} \mathrm{P}<0.001$ vs. miR-206 inhibited hFOB1.19 cells. NC, negative control; PDCD4, programmed cell death 4; ALP, alkaline phosphatase; Bcl-2, B-cell lymphoma 2; Bax, Bcl-2-X-associated protein; miR, microRNA; si, small interfering; FITC, fluorescein isothiocyanate; PI, propidium iodide.

miR-26a and miR-196 are miR members that serve central roles in the osteogenic differentiation of mesenchymal stem cells (28-30). Additionally, miR-29, miR-let-7 and miR-26 attenuate extracellular matrix protein synthesis and mineralization in osteoblasts as well as support the maturation of osteoblasts (10). In the present study, the expression of miR-206 was first investigated in clinical SANFH specimens. Consistent with a previous study (17), it was demonstrated that the expression of miR-206 was associated with the onset of SANFH. A previous study demonstrated that the overexpression of miR-206 inhibits the differentiation of osteoblasts via the $\mathrm{Wnt} / \beta$-catenin signaling pathway (17). With a series of in vitro assays, the present study confirmed the negative effect miR-206 on viability and differentiation potential on osteoblasts. Induced expression of miR-206 not only increased the apoptotic rate but also inhibited the cell viability of hFOB1.19 cells. In addition, the expression of the osteoblastic differentiation marker ALP was also inhibited by the miR-206 mimic. To investigate the downstream signaling involved in the effect of miR-206 on osteoblasts, the function of PDCD4 was also investigated in the present study. PDCD4 is 
originally characterized as an inhibitor of cellular transformation (31). In several cancer types, the expression of PDCD4 is inhibited $(32,33)$. In the study of Sugatani et al (22) the authors confirm a novel molecular mechanism regulating osteoclastogenesis: enforced expression of miR-21 inhibited osteoclast development via down-regulation of PDCD4 expression. The conclusion infers a pro-osteoblastogenic role for PDCD4, which was validated by the results of the present study. Similar to the effect of increased miR-206 expression, knockdown of PDCD4 induced apoptosis and impaired the differentiation potential of hFOB1.19 cells, which inhibited the pro-osteoblastogenesis effect of miR-206 inhibition.

In conclusion, the results of the present study supplement the knowledge about the function of miR-206 in the onset and progression of SANFH. miR-206 induced the apoptosis and suppressed proliferation and differentiation of osteoblasts in a PDCD4-dependent manner. Modulation of miR-206 signaling will be an innovative method for alleviating the deleterious effect of SANFH. Our results support the potential of miR-206 to be used in therapies for the treatment of SANFH but further studies need to be conducted.

\section{Acknowledgements}

This work is supported by Science and Technology Department of Guangdong Province (2014A020212041) and Shenzhen Science and Technology Innovation Committee (JCYJ20140411091151442).

\section{References}

1. Herring JA: Management of Perthes' disease. J Pediatr Orthop 16: $1-2,1996$

2. Beckmann R, Shaheen H, Kweider N, Ghassemi A, Fragoulis A, Hermanns-Sachweh B, Pufe T, Kadyrov M and Drescher W: Enoxaparin prevents steroid-related avascular necrosis of the femoral head. ScientificWorldJournal 2014: 347813, 2014.

3. Nishida K, Yamamoto T, Motomura G, Jingushi S and Iwamoto Y: Pitavastatin may reduce risk of steroid-induced osteonecrosis in rabbits: A preliminary histological study. Clin Orthop Relat Res 466: 1054-1058, 2008.

4. Takano-Murakami R, Tokunaga K, Kondo N, Ito T, Kitahara H, Ito $\mathrm{M}$ and Endo $\mathrm{N}$ : Glucocorticoid inhibits bone regeneration after osteonecrosis of the femoral head in aged female rats Tohoku J Exp Med 217: 51-58, 2009.

5. Samara S, Dailiana Z, Chassanidis C, Koromila T, Papatheodorou L, Malizos KN and Kollia P: Expression profile of osteoprotegerin, RANK and RANKL genes in the femora head of patients with avascular necrosis. Exp Mol Pathol 96: 9-14, 2014.

6. Weinstein RS, Jilka RL, Parfitt AM and Manolagas SC: Inhibition of osteoblastogenesis and promotion of apoptosis of osteoblasts and osteocytes by glucocorticoids. Potential mechanisms of their deleterious effects on bone. J Clin Invest 102: 274-282, 1998.

7. Kerachian MA, Séguin C and Harvey EJ: Glucocorticoids in osteonecrosis of the femoral head: A new understanding of the mechanisms of action. J Steroid Biochem Mol Biol 114: 121-128, 2009.

8. Yun SI, Yoon HY, Jeong SY and Chung YS: Glucocorticoid induces apoptosis of osteoblast cells through the activation of glycogen synthase kinase 3beta. J Bone Miner Metab 27: 140-148, 2009.

9. Kim SJ, Bahk WJ, Chang CH, Jang JD and Suhl KH: Treatment of osteonecrosis of the femoral head using autologous cultured osteoblasts: A case report. J Med Case Rep 2: 58, 2008.

10. van Wijnen AJ, van de Peppel J, van Leeuwen JP, Lian JB, Stein GS, Westendorf JJ, Oursler MJ, Im HJ, Taipaleenmäki H, Hesse E, et al: MicroRNA functions in osteogenesis and dysfunctions in osteoporosis. Curr Osteoporos Rep 11: 72-82, 2013.
11. Lian JB, Stein GS, van Wijnen AJ, Stein JL, Hassan MQ, Gaur T and Zhang Y: MicroRNA control of bone formation and homeostasis. Nat Rev Endocrinol 8: 212-227, 2012.

12. Miyaki S and Asahara H: Macro view of microRNA function in osteoarthritis. Nat Rev Rheumatol 8: 543-552, 2012.

13. Xia Z, Chen C, Chen P, Xie H and Luo X: MicroRNAs and their roles in osteoclast differentiation. Front Med 5: 414-419, 2011.

14. Goldring MB and Marcu KB: Epigenomic and microRNA-mediated regulation in cartilage development, homeostasis, and osteoarthritis. Trends Mol Med 18: 109-118, 2012.

15. Taipaleenmäki H, Bjerre Hokland L, Chen L, Kauppinen S and Kassem M: Mechanisms in endocrinology: micro-RNAs: Targets for enhancing osteoblast differentiation and bone formation. Eur J Endocrinol 166: 359-371, 2012

16. Inose H, Ochi H, Kimura A, Fujita K, Xu R, Sato S, Iwasaki M, Sunamura S, Takeuchi Y, Fukumoto S, et al: A MicroRNA regulatory mechanism of osteoblast differentiation. Proc Natl Acad Sci USA 106: 20794-20799, 2009.

17. Liu G, Luo G, Bo Z, Liang X, Huang J and Li D: Impaired osteogenic differentiation associated with connexin43/microRNA-206 in steroid-induced avascular necrosis of the femoral head. Exp Mol Pathol 101: 89-99, 2016.

18. Krichevsky AM and Galina G: miR-21: A small multi-faceted RNA. J Cell Mol Med 13: 39-53, 2009.

19. Loh PG, Yang HS, Walsh MA, Wang Q, Wang X, Cheng Z, Liu D and Song H: Structural basis for translational inhibition by the tumour suppressor Pdcd4. EMBO J 28: 274-285, 2009.

20. Livak KJ and Schmittgen TD: Analysis of relative gene expression data using real-time quantitative PCR and the 2(-Delta Delta C(T)) method. Methods 25: 402-408, 2001

21. Yu H, Shi L, Qi G, Zhao S, Gao Y and Li Y: Gypenoside protects cardiomyocytes against ischemia-reperfusion injury via the inhibition of mitogen-activated protein kinase mediated nuclear factor kappa B pathway in vitro and in vivo. Front Pharmacol 7: 148, 2016.

22. Sugatani T, Vacher J and Hruska KA: A microRNA expression signature of osteoclastogenesis. Blood 117: 3648-3657, 2011.

23. Cheng JC, Lam TP and Ng BK: Prognosis and prognostic factors of Legg-Calve-Perthes disease. J Pediatr Orthop 31 (2 Suppl): S147-S151,2011.

24. Ito H, Matsuno T and Kaneda K: Prognosis of early stage avascular necrosis of the femoral head. Clin Orthop Relat Res: 149-157, 1999.

25. Ohzono K, Saito M, Takaoka K, Ono K, Saito S, Nishina T and Kadowaki T: Natural history of nontraumatic avascular necrosis of the femoral head. J Bone Joint Surg Br 73: 68-72, 1991.

26. Hao C, Yang S, Xu W, Shen JK, Ye S, Liu X, Dong Z, Xiao B and Feng Y: MiR-708 promotes steroid-induced osteonecrosis of femoral head, suppresses osteogenic differentiation by targeting SMAD3. Sci Rep 6: 22599, 2016.

27. Bai R, Feng W, Liu WL, Zhao ZH, Zhao AQ, Wang Y, Wang WX, Sun L, Wu LS and Cui SH: Roles of osteocyte apoptosis in steroid-induced avascular necrosis of the femoral head. Genet Mol Res, 15, 2016

28. Meng YB, Li X, Li ZY, Zhao J, Yuan XB, Ren Y, Cui ZD, Liu YD and Yang XJ: microRNA-21 promotes osteogenic differentiation of mesenchymal stem cells by the PI3K/ $\beta$-catenin pathway. J Orthop Res 33: 957-964, 2015.

29. Su X, Liao L, Shuai Y, Jing H, Liu S, Zhou H, Liu Y and Jin Y. MiR-26a functions oppositely in osteogenic differentiation of BMSCs and ADSCs depending on distinct activation and roles of Wnt and BMP signaling pathway. Cell Death Dis 6: e1851, 2015.

30. Candini O, Spano C, Murgia A, Grisendi G, Veronesi E, Piccinno MS, Ferracin M, Negrini M, Giacobbi F, Bambi F, et al: Mesenchymal progenitors aging highlights a miR-196 switch targeting HOXB7 as master regulator of proliferation and osteogenesis. Stem Cells 33: 939-950, 2015.

31. Jansen AP: A novel transformation suppressor, Pdcd4, inhibits AP-1 transactivation but not NF-kappaB or ODC transactivation. Oncogene 20: 669-676, 2001.

32. Jansen AP, Camalier CE, Stark C and Colburn NH: Characterization of programmed cell death 4 in multiple human cancers reveals a novel enhancer of drug sensitivity. Mol Cancer Ther 3: 103-110, 2004.

33. Göke R, Barth P, Schmidt A, Samans B and Lankat-Buttgereit B: Programmed cell death protein 4 suppresses CDK1/cdc2 via induction of p21 (Waf1/Cip1). Am J Physiol Cell Physiol 287: C1541-C1546, 2004.

This work is licensed under a Creative Commons Attribution-NonCommercial-NoDerivatives 4.0 International (CC BY-NC-ND 4.0) License. 